\title{
Changes In The Size Of Capillaries In Rabbit Skeletal Muscle Following Deep Transverse Friction and Compressed Air Massage
}

*M.A. Gregory, ${ }^{\wedge}$ M.Mars and ** M.N.Deane

*Electron Microscope Unit, and **Department of Physiotherapy, University of Durban-Westville ${ }^{\wedge}$ Physiology Dept., Nelson R. Mandela Medical School, University of Natal, Durban, South Africa

Key objectives when using massage to treat soft tissue lesions are to encourage damaged tissue to rapidly regain tensile strength and for lesions to heal with a flexible, functional scar. The precise mechanisms by which massage promotes such repair are unknown. Various authors have attributed the beneficial effects of massage to local manipulative effects on blood vessels causing an increase in temperature and elevated skin and muscle blood flow[1]. In this study we investigate this premise by recording changes in the diameters of capillaries in rabbit skeletal muscle up to 6 days following deep transverse friction massage (DTF), a therapy that provides therapeutic movement over a small area, and compressed air massage (JET $\left.{ }^{\circledR}\right)$ a treatment that manipulates a wider area.

Twenty four New Zealand, white rabbits were randomly divided into 2 groups to be treated by DTF (R1 - R12) or JET (R13 - R24) Before either treatment, the animals were anaesthetised by an intramuscular injection of equal parts Ketamine and Xylazine $(10 \mathrm{mg} / \mathrm{kg})$. The fur was removed from the skin at the level of the mid-thigh. To ensure that DTF was applied to the same area, the treatment site was clearly marked with indelible ink. Treatment was a single, 10 minute episode of DTF using the index finger or 10 minutes of JET therapy using the "sport", single hole $(4 \mathrm{~mm})$ head and unheated air at 1 bar. Following treatment, wedge biopsies approximately $0.5 \mathrm{~cm} \mathrm{X} 1 \mathrm{~cm}$ were taken from either the left biceps femoris (DTF) or left vastus lateralis (JET) within 10 minutes (R1 - R4: R13 - R16), 24 hours (R5 - R8: R17 - 20) and 6 days after treatment (R9 - R12: R21 - 24). To serve as controls, similar biopsies were taken from the right biceps femoris or vastus lateralis of animals R9 - R12 and R21 - 24. The samples were immediately placed in Karnovsky's fixative in $0.2 \mathrm{M}$ cacodylate buffer and blocks of tissue approximately $1 \mathrm{~mm} \mathrm{X} 2 \mathrm{~mm}$ excised in such a way that the greater dimension followed the longitudinal axis of muscle bundles. Following dehydration and perfusion with Spurr's epoxy resin, the tissue was longitudinally orientated and "flat" embedded in silicone moulds. Thick, $(1 \mu \mathrm{m})$ transverse sections of muscle were cut and stained with $1 \%$ aqueous alkaline toluidine blue. Sections were examined with an LM using a X40 objective and images of areas of interest captured using an image analyser with SIS software (Fig. 1). The cross-sectional diameters of a minimum 60 capillaries were measured from each specimen. Statistical analyses was by Kruskal-Wallace test with Dunn's post hoc multiple comparisons test.

The results showed that mean capillary diameters varied in different muscles groups in close proximity to one another. Capillaries perfusing vastus lateralis muscle were significantly larger ( $\mathrm{p}$ $<0.01)$ than those in their biceps femoris counterparts. The data confirmed that massage, per-se, caused a rapid and significant increase of capillary diameters in rabbit skeletal muscle (Table). It also showed that different types of massage had a more or less prolonged effect on capillary dilatation (Fig. 2). In the case of DTF, there was an immediate significant increase of $18 \%$ in crosssectional area reducing to $9 \%$ after $24 \mathrm{hrs}$ and $-7 \% 6$ days later. JET therapy caused an immediate increase of $13 \%$ which rose to $22 \%$ after 24 hours before falling to a $4 \%$ increase after 6 days. 
The difference of control capillary diameters could be a function of the different types of muscle: biceps femoris being slow twitch and vastus lateralis fast twitch muscle. The difference in the pattern of extended capillary dilatation is probably a consequence of the different types of massage therapy. In the case of DTF, focussed pressure causes a local increase in skin and subcutaneous temperature. JET therapy causes a more diffuse manipulation over a wider area with the passage of air cooling both the skin and subcutaneous tissues[2]. While the data shows significant and varying increases in the diameter and cross-sectional area of capillary's after massage, the resolution of the light microscope does not enable the (patho)-morphological state of endothelial cells nor the diameter/area of the lumen to be determined. Without such information, it is not possible to confirm the probability of improved blood flow after massage. A more precise evaluation of this phenomenon using TEM and ultrastructural morphometry is the subject of ongoing investigations.

\section{References}

[1] D.J. Wyper and D.R. McGiven. Physiotherapy 62 (1976)83.

[2] M. Mars. S. Afr. J. Sports Med. 8, 3 (2001) 30.

\begin{tabular}{|l|r|r|r|r|r|r|r|r|}
\hline \multicolumn{7}{|c|}{ DTF Therapy } & \multicolumn{3}{|c|}{ JET Therapy } \\
\hline & Control & R1-R4 & R5-R8 & R9-R12 & Control & R13-R16 & R17-R20 & R21-R24 \\
\hline $\mathbf{n}$ & 342 & 272 & 426 & 412 & 416 & 402 & 355 & 488 \\
Mean-D & $\mathbf{4 . 7 6}$ & $\mathbf{5 . 2}$ & $\mathbf{5 . 0}$ & $\mathbf{4 . 6}$ & $\mathbf{5 . 1}$ & $\mathbf{5 . 3}$ & $\mathbf{5 . 5}$ & $\mathbf{5 . 2}$ \\
SD & 1.28 & 1.3 & 1.4 & 1.1 & 1.1 & 1.2 & 1.5 & 1.2 \\
Er.Mean & & & & & 0.05 & 0.06 & 0.08 & 0.06 \\
High & 10.94 & 12.3 & 12.5 & 11.5 & 9.0 & 12.0 & 14.0 & 11.0 \\
Low & 2.33 & 2.2 & 2.4 & 2.3 & 2.9 & 2.9 & 2.1 & 2.5 \\
Area & $\mathbf{1 7 . 8 2}$ & $\mathbf{2 1 . 1}$ & $\mathbf{1 9 . 4}$ & $\mathbf{1 6 . 6}$ & $\mathbf{2 0 . 4}$ & $\mathbf{2 2 . 1}$ & $\mathbf{2 3 . 8}$ & $\mathbf{2 1 . 2}$ \\
\hline Cont.p $=$ & & $<0.01$ & $<0.01$ & $>0.05$ & & $<0.01$ & $<0.001$ & $>0.05$ \\
\hline
\end{tabular}

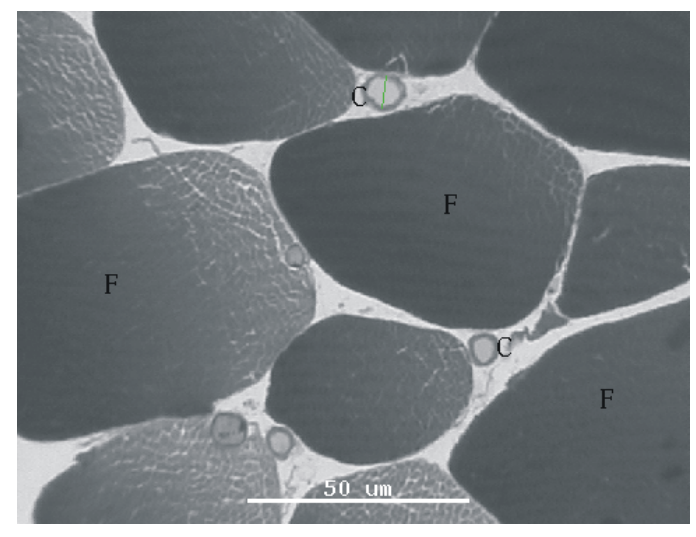

Fig. 1

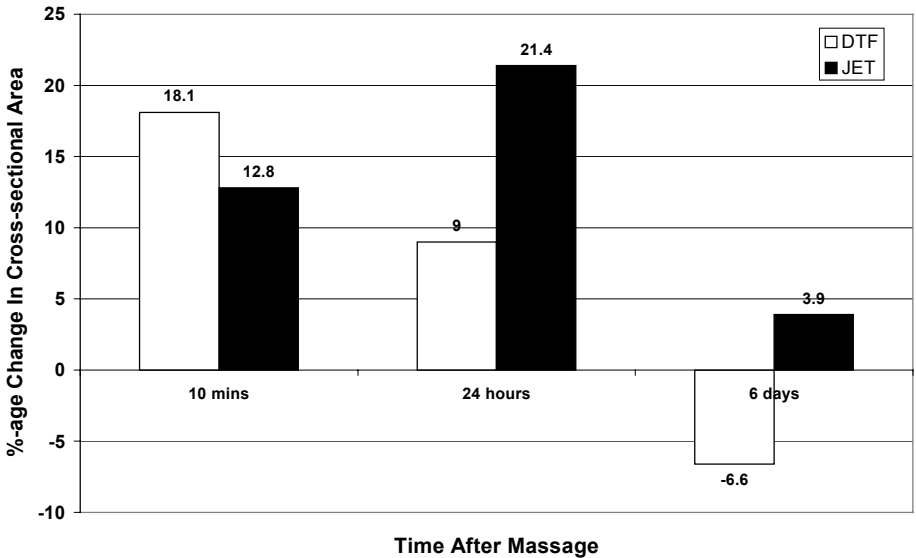

F ig. 2

Table: Summary of morphometric data from control and post-DTF \& JET treated muscle. $\mathrm{n}=$ number of measurements; Mean-D = group average capillary diameters of group $(\mu \mathrm{m})$; $\mathrm{SD}=$ standard deviation; EM = error of the mean; Cont.p = significance of difference between data when compared with control.

Fig. 1: Light micrograph showing cross-sectioned capillaries and measurement positions.

Fig. 2: Shows the percentage change in the cross-sectional area of capillaries after DTF or compressed air massage. 